\title{
Photometric Analysis of the Pi of the Sky Data
}

\author{
M. Siudek, K. Malek, L. Mankiewicz, R. Opiela, M. Sokolowski, A. F. Żarnecki
}

\begin{abstract}
A database containing star measurements from the period 2006-2009 taken by the Pi of the Sky detector located in Las Campanas Observatory in Chile contains more than 2 billion measurements of almost 17 million objects. All measurements are available on the $\mathrm{Pi}$ of the Sky web site through a dedicated interface, which also allows users to download selected data. Accurate analysis of Pi of the Sky data is a real challenge, because of a number of factors that can influence the measurements. Possible sources of errors in our measurements include: reading the chip with the shutter open, strong and varying sky background, passing planets or planetoids, and clouds and hot pixels. In order to facilitate the analysis of variable stars we have developed a system of dedicated filters to remove bad measurements or frames. The spectral sensitivity of the detector is taken into account by appropriate corrections based on the spectral type of reference stars. This process is illustrated by an analysis of the BG Ind system, where we have been able to reduce the systematic uncertainty to about 0.05 magnitudo.
\end{abstract}

Keywords: Gamma Ray Burst (GRB), prompt optical emissions, optical flashes, nova stars, variable stars, robotic telescopes, photometry.

\section{Introduction}

$\mathrm{Pi}$ of the Sky [4] is a robotic telescope aimed at monitoring a large part of the sky with a good range and time resolution. The detector was designed for observations of astrophysical phenomena characterized by short timescales, especially for prompt optical counterparts of Gamma Ray Bursts (GRBs). The Pi of the Sky apparatus design and observational strategy make this detector useful also for searching for nova and supernova stars and for monitoring interesting objects such as blasars, AGNs and variable stars.

The full Pi of the Sky system will consist of 2 sites separated by a distance of about $100 \mathrm{~km}$, allowing flashes from satellites and other near-Earth objects to be rejected. Each site will consist of 12 customdesigned CCD cameras placed on specially designed equatorial mounts (4 cameras per mount). The full system is now under construction.

Necessary tests before constructing the final version were performed with a prototype consisting of 2 custom-designed cameras placed on an equatorial mount. The prototype operated at the Las Campanas Observatory in Chile from June 2004 till the end of 2009. Cameras working in coincidence observed a $20^{\circ} \times 20^{\circ}$ field of view with a time resolution of 10 seconds. Each camera is equipped with Canon lenses $f=85 \mathrm{~mm}, d=f / 1.2$, which allows observation of objects to about $12^{m}$ (about $13.5^{m}$ for 20 coadded frames). During the 6 years of work the prototype gathered a large amount of data valuable

\footnotetext{
${ }^{1} \mathrm{Pi}$ of the Sky Home Page: http://grb.fuw.edu.pl/pi/
}

for astronomical research, e.g. for identifying and cataloging many different types of variable stars [2].

\section{Pi of the Sky databases}

All data gathered by the Pi of the Sky detector are stored in publicly accessible databases ${ }^{1}$. Currently three databases are available: the first database, covering the period from June 2004 till May 2005, contains almost 800 million measurements for about 4.5 million objects. The second database, from May 2006-November 2007, is a subset of the third database, which contains data gathered from May 2006-April 2009. The third database is the biggest, and includes about 2.16 billion measurements for about 16.7 million objects. In order to ensure easy access to our measurements, we have developed a user-friendly web interface [1]. It allows stars to be selected according to their type, magnitudo, coordinates, etc, displaying their light curves and other properties. It is also possible to download large packets of light curves of multiple stars.

\section{Improving photometry}

\subsection{System of dedicated filters}

The data acquired during the $\mathrm{Pi}$ of the Sky observations is reduced by a fully automatic pipeline, and only the light curves of stars are stored in the database. In many aspects of analysis, e.g. identification of variable stars, it is important to select only 

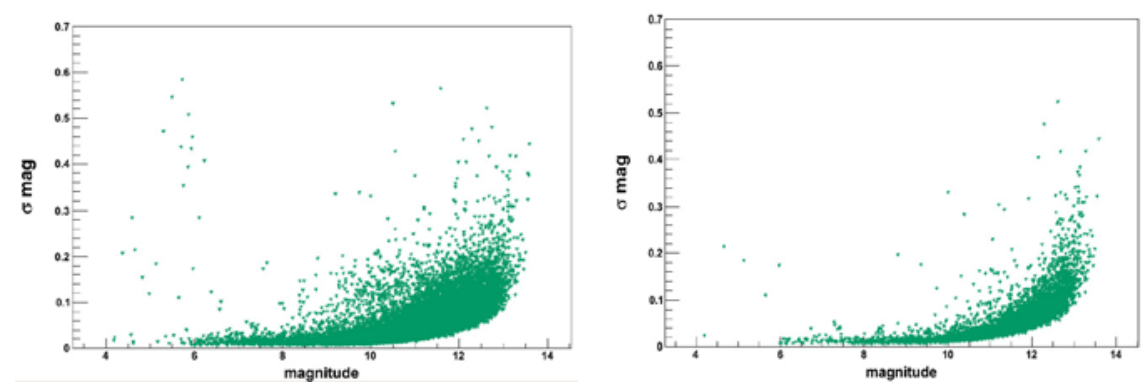

Fig. 1: Precision dispersion of star brightness measurements from standard photometry for 200s exposures (20 coadded frames) from the Pi of the Sky prototype at the Las Campanas Observatory in Chile. Large dispersion (left) is mainly caused by false measurements. After the application of quality cuts (right), the photometry accuracy improves significantly

data with high measurement precision. In order to fulfill this requirement we have developed a system of data quality cuts. The system of dedicated filters allows the rejection of measurements or even whole frames affected by detector imperfections or observation conditions. Measurements that are placed near the border of the frame, or that are infected by hot pixels, bright background caused by an open shutter or moon halo, or by planet or planetoid passage, can easily be excluded from further analysis. Filters allowing the removal of measurements infected by one of these effects are available on the web interface.

Applying quality cuts can significantly improve photometry accuracy (see Figure 1). For stars with range $7-10^{m}$, average photometry uncertainty sigma of about $0.018-0.024^{m}$ has been achieved.

\subsection{Approximate color calibration algorithm}

The Pi of the Sky detector is characterized by relatively wide spectral sensitivity due to the fact that the observations are performed only with an IR-UVcut filter. The average wavelength is about $585 \mathrm{~nm}$ and corresponds to a $\mathrm{V}$ filter, which is also used as a reference in photometry corrections. It turned out that the detector response is correlated with the star spectral type. The average magnitudo measured by $\mathrm{Pi}$ of the Sky $\left(M_{P I}\right)$ is shifted with respect to the difference of the catalogue magnitudo given by $\mathrm{B}-\mathrm{V}$ or $\mathrm{J}-\mathrm{K}$. Approximativy of this dependence with a linear function enables the measurement of each star to be corrected. After applying the correction, the distribution of the average magnitudo shift for the reference stars becomes significantly narrower (an example of the reference stars for BG Ind variable [3] is shown in Figure 2).

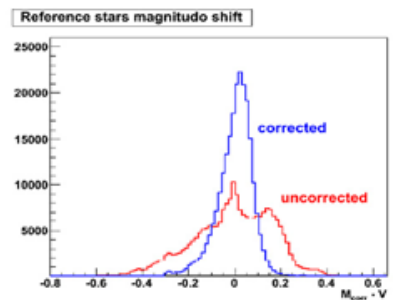

Fig. 2: Distribution of the average magnitudo shift for reference stars after standard photometry (red) and after spectral correction (blue)

Further conditions, including maximal accepted shift, RMS of $M_{\text {coor }}$ and number of measurements, are used to evaluate additional photometry corrections (see Figure 3).
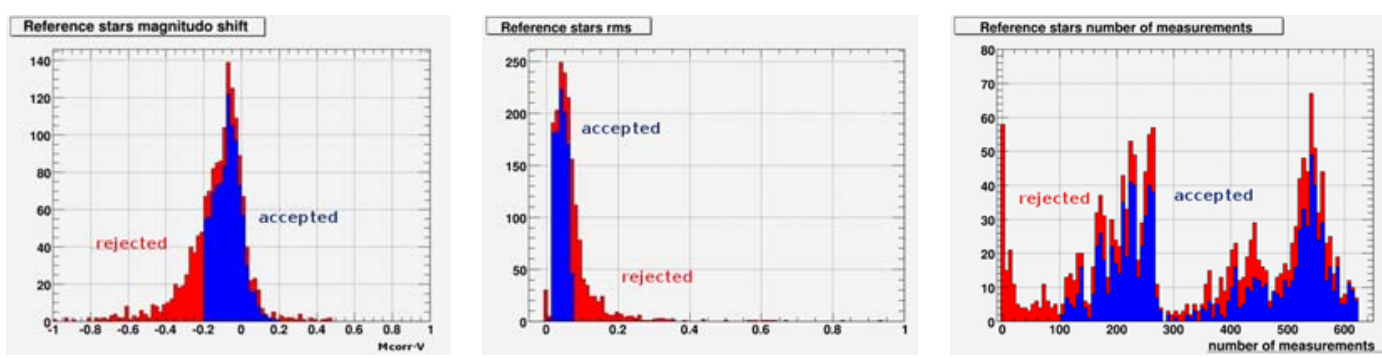

Fig. 3: For calculating photometry corrections, only the best reference stars were used (blue) after rejecting stars with magnitudo shift $\left(M_{\text {coor }}-V\right)$ bigger than $0.2, R M S_{\text {coor }}$ bigger than 0.07 and with number of measurements smaller than 100 (red) 
Significant improvement of measurement precision is also achieved when the photometry correction is not calculated as a simple average over all selected reference stars, but when a quadratic dependence of the correction on the reference star position in the sky is fitted for each frame. $\chi^{2}$ distribution can be used to select measurements with the most precise photometry (see Figure 4).

The effect of photometry correction with a distribution of $\chi^{2}$ on the reconstructed BG Ind light curve is shown in Figure 5.

Applying the new algorithm improved the photometry quality, and uncertainty sigma of the order of $0.013^{m}$ was obtained [3]. We also applied photometry correction to other stars, with as good results as in the case of BG Ind variable. Spectral correction and additional $\chi^{2}$ distribution allow the selection of only the measurements with the highest precision.
With the example of cepheid variable, T Cru and GH Car we can see that the light curve is significantly improved and the measurements flagged as those with potentially worse precision do indeed tend to stand outside the light curve (see Figure 6).

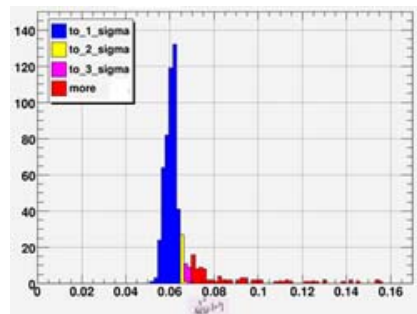

Fig. 4: $\chi^{2}$ distribution. For about $20 \%$ of the calculated frames, $\chi^{2}$ is greater than 0.058. This information can be used to select measurements with the most precise photometry
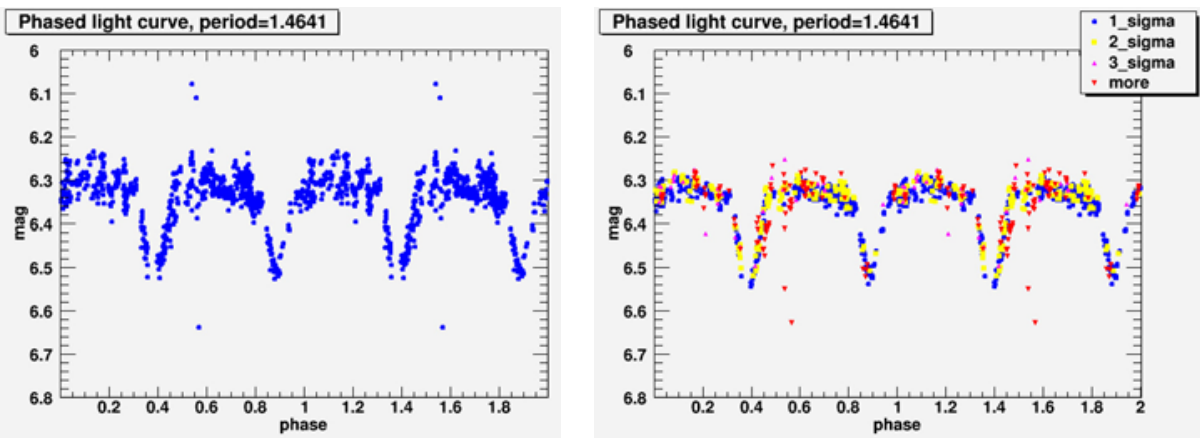

Fig. 5: Uncorrected light curve for BG Ind variable (left) and after spectral corrections with correction quality cut (right)
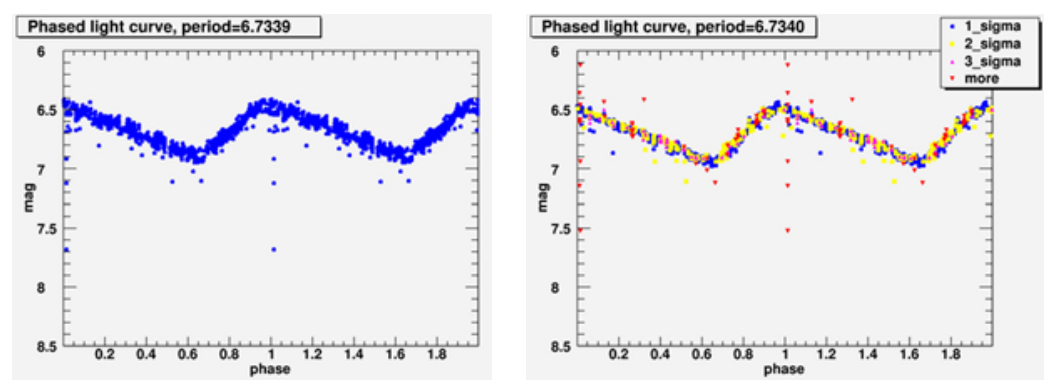

Fig. 6: Uncorrected light curve for T Cru variable (left) and after spectral corrections with correction quality cut (right)
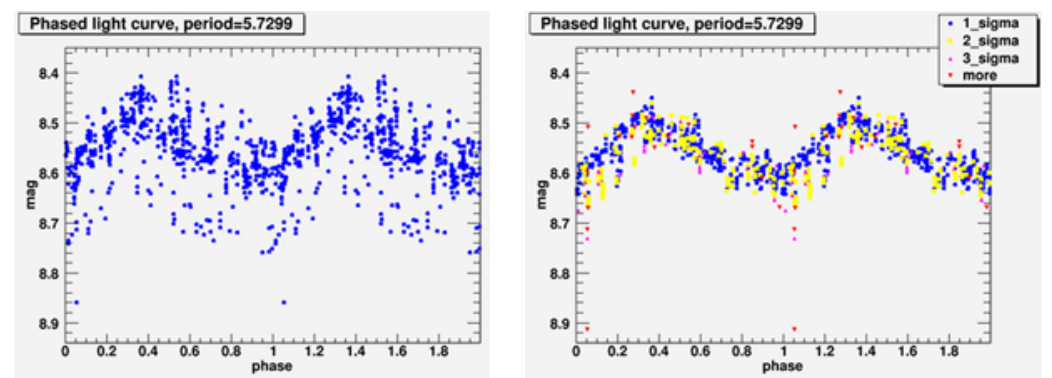

Fig. 7: Uncorrected light curve for GH Car variable (left) and after spectral corrections with correction quality cut (right) 


\section{Summary}

During the period 2006-2009 the prototype observed almost 17 million objects and collected over 2 billion measurements. The data acquired by $\mathrm{Pi}$ of the Sky is publicly available through a user-friendly web interface. In order to facilitate selection of measurements with high precision, we have developed a system of dedicated filters to remove measurements affected by detector imperfections or observation conditions. When an approximate color calibration algorithm based on the spectral type of reference stars is used, an uncertainty sigma of the order of $0.013^{m}$ can be obtained.

\section{Acknowledgement}

We are very grateful to G. Pojmanski for access to the ASAS dome and for sharing his experience with us. We would like to thank the staff of the Las Campanas Observatory, San Pedro de Atacama Observatory and the INTA El Arenosillo test centre in Mazagón near Huelva for their help during installation and maintenance of our detector. This work was financed by the Polish Ministry of Science and Higher Education in 2009-2011 as a research project.

\section{References}

[1] Biskup, M., et al.: Web interface for star databases of the Pi of the Sky Experiment, Proceedings of SPIE, Vol. 6 937, 2007.

[2] Majczyna, A., et al.: Pi of the sky catalogue of the variable stars from 2006-2007 data, Proceedings of SPIE, Vol. $\mathbf{7} \mathbf{7 4 5}, 2010$.

[3] Rozyczka, M., Malek, K., et al.: Absolute properties of BG Ind - a bright F3 system just leaving the Main Sequence, Monthly Notices of the Royal Astronomical Society, 2011, 414, 2479-2 485.

[4] Siudek, M., et al.: Pi of the Sky telescopes in Spain and Chile, in these proceedings.

Malgorzata Siudek

Katarzyna Malek

Lech Mankiewicz

Rafal Opiela

Center for Theoretical Physics

of the Polish Academy of Sciences

Al. Lotnikow 32/46, 02-668 Warsaw, Poland

Marcin Sokolowski

The Andrzej Soltan Institute for Nuclear Studies Hoza 69, 00-681 Warsaw, Poland

Aleksander F. Żarnecki

Faculty of Physics

University of Warsaw

Hoza 69, 00-681 Warsaw, Poland 